\title{
Multiple solutions for superlinear double phase Neumann problems
}

\author{
Nikolaos S. Papageorgiou' • Vicenţiu D. Rădulescu²,3 - Youpei Zhang ${ }^{3,4}$
}

Received: 21 July 2021 / Accepted: 27 September 2021

(c) The Author(s) 2022

\section{Abstract}

We study a double phase Neumann problem with a superlinear reaction which need not satisfy the Ambrosetti-Rabinowitz condition. Using the Nehari manifold method, we show that the problem has at least three nontrivial bounded ground state solutions, all with sign information (positive, negative and nodal).

Keywords Double phase - Musielak-Orlicz-Sobolev · Constant sign and nodal solutions · Nehari manifold $\cdot$ Superlinear reaction

Mathematics Subject Classification 35J75 (Primary); 35A16 - 35B50 - 35B51 - 35J20 . 35J60 $\cdot 47 \mathrm{~J} 15 \cdot 58 \mathrm{E} 05 \cdot 58 \mathrm{E} 07$ (Secondary)

\section{Introduction}

Let $\Omega \subseteq \mathbb{R}^{N}$ be a bounded domain with a Lipschitz boundary $\partial \Omega$. In this paper we study the following double phase Neumann problem

$$
\left\{\begin{array}{l}
-\Delta_{p}^{a} u(z)-\Delta_{q} u(z)+\xi(z)|u(z)|^{q-2} u(z)=f(z, u(z)) \text { in } \Omega, \\
\frac{\partial u}{\partial n}=0 \text { on } \partial \Omega, 1<q<p<N .
\end{array}\right\}
$$

凶Vicenţiu D. Rădulescu

radulescu@inf.ucv.ro

Nikolaos S. Papageorgiou

npapg@math.ntua.gr

Youpei Zhang

zhangypzn@163.com; youpei.zhang@inf.ucv.ro

1 Department of Mathematics, Zografou Campus, National Technical University, Athens 15780, Greece

2 Faculty of Applied Mathematics, AGH University of Science and Technology, al. Mickiewicza 30, 30-059 Kraków, Poland

3 School of Mathematics and Statistics, Central South University, Changsha, Hunan 410083, China

4 Department of Mathematics, University of Craiova, Street A.I. Cuza No. 13, Craiova 200585, Romania 
Given $a \in L^{\infty}(\Omega) \backslash\{0\}$ with $a(z) \geq 0$ for a.a. $z \in \Omega$ and $1<r<+\infty$, we denote by $\Delta_{r}^{a}$ the weighted $r$-Laplace differential operator defined by

$$
\Delta_{r}^{a} u=\operatorname{div}\left(a(z)|D u|^{r-2} D u\right) .
$$

If $a \equiv 1$, then we have the usual $r$-Laplacian denoted by $\Delta_{r}$. The differential operator in problem (1) is the sum of two such operators with different exponents. Hence the differential operator is not homogeneous. There is also a potential term $\xi(z)|u|^{q-2} u$ with $\xi \in L^{\infty}(\Omega) \backslash\{0\}$, $\xi(z) \geq 0$ for a.a. $z \in \Omega$. The differential operator of problem (1) is related to the so-called "double phase" integral functional defined by

$$
\int_{\Omega}\left(a(z)|D u|^{p}+|D u|^{q}\right) d z .
$$

The integrand of this functional is

$$
\theta(z, x)=a(z) x^{p}+x^{q} \text { for all } z \in \Omega, \text { all } x \geq 0 .
$$

We do not assume that the weight $a(\cdot)$ is bounded away from zero (that is, we do not have that $\left.\operatorname{ess~inf}_{\Omega} a>0\right)$. Therefore the integrand $\theta(z, x)$ exhibits unbalanced growth, namely

$$
x^{q} \leq \theta(z, x) \leq c_{0}\left(x^{q}+x^{p}\right) \text { for a.a. } z \in \Omega \text {, all } x \geq 0 \text {, some } c_{0}>0 .
$$

Such functionals, wre first considered by Marcellini [14,15] and Zhikov [27,28], in the context of problems of the calculus of variations and of nonlinear elasticity theory. Recently the interest for such problems was revived and some important interior regularity results were obtained for local minimizers of such functionals. We refer to the works of Baroni et al. [1], Mingione and Rădulescu [16], and Ragusa and Tachikawa [24]. However, until now there is no global regularity theory (that is, regularity results up to the boundary) for the solutions of such problems. This fact limits the tools available for the study of such equations and makes double phase problems more difficult to deal with. In fact, the unbalanced growth of $\theta(z, \cdot)$ implies that the usual Sobolev spaces do not suffice for the analysis of the problem and we have to use the general abstract setting of Musielak-Orlicz-Sobolev spaces.

In the reaction of problem (1), we have a Carathéodory function $f(z, x)$ (that is, for all $x \in \mathbb{R}$ the mapping $z \mapsto f(z, x)$ is measurable and for a.a. $z \in \Omega$ the function $x \mapsto f(z, x)$ is continuous). We assume that $f(z, \cdot)$ exhibits $(p-1)$-superlinear growth as $x \rightarrow \pm \infty$ but without satisfying the usual in such cases Ambrosetti-Rabinowitz condition (the ARcondition for short). The absence of a global regularity theory for such problems requires a different approach for double phase equations. In problems with balanced growth $((p, q)$ equations), for which a powerful global regularity theory exists (see Lieberman [10]), the main tools are truncation and comparison techniques, critical point theory and Morse theory (critical groups). We refer to the works of Liu and Papageorgiou [12], Papageorgiou and Rădulescu [17,18], Papageorgiou et al. [21], Papageorgiou and Zhang [23]. Here instead, we use the Nehari manifold method as this was developed by Brown and $\mathrm{Wu}$ [2], Brown and Zhang [3], Szulkin and Weth [25] and Willem [26].

We show that problem (1) has at least three nontrivial bounded solutions, all with sign information (positive, negative and nodal (sign changing)). Recently there have been some multiplicity results for double phase problems. We refer to the works of Gasiński and Papageorgiou [5], Ge et al. [8], Liu and Dai [11], Papageorgiou et al. [22] (Dirichlet problems) and Gasiński and Winkert [7], Papageorgiou, Rădulescu and Repovš [20] (Neumann and Robin problems). Closer to our work here are the papers of Gasiński and Papageorgiou [5] and Liu and Dai [11], which deal with superlinear Dirichlet problems, employing more restrictive conditions on the reaction and using a different approach. 


\section{Mathematical background and hypotheses}

As we already mentioned in the introduction, in order to accommodate the unbalanced growth of $\theta(z, \cdot)$, we have to use Musielak-Orlicz-Sobolev spaces. A comprehensive presentation of the theory of these spaces can be found in the recent book of Harjulehto and Hästö [9].

We make the following hypotheses on the weight $a(\cdot)$, the potential function $\xi(\cdot)$ and exponents $q, p$.

$\mathrm{H}_{0}: a \in C^{0,1}(\bar{\Omega})$ (that is, $a: \bar{\Omega} \mapsto \mathbb{R}$ is Lipschitz continuous), $a(z)>0$ for all $z \in \Omega$, $\xi \in L^{\infty}(\Omega) \backslash\{0\}, \xi(z) \geq 0$ for a.a. $z \in \Omega, 1<q<p<q^{*}\left(q^{*}=\frac{N q}{N-q}\right), p<N$.

Remark 1 In contrast to previous works on Dirichlet double phase problems, we do not require that $\frac{p}{q}<1+\frac{1}{N}$. This condition implies that $p<q^{*}$, but the converse is not true. For Dirichlet problems this condition guarantees that the Poincaré inequality is valid for the corresponding Musielak-Orlicz-Sobolev space (see Harjulehto \& Hästö [9, pp. 100, 138]).

Let $\mathrm{M}(\Omega)$ be the space of all Lebesgue measurable functions $u: \Omega \mapsto \mathbb{R}$. As usual we identify two measurable functions which differ only on a Lebesgue-null subset of $\Omega$. Then the Musielak-Orlicz space $L^{\theta}(\Omega)$ is defined by

$$
L^{\theta}(\Omega)=\left\{u \in \mathrm{M}(\Omega): \rho_{\theta}(u)<+\infty\right\},
$$

with $\rho_{\theta}(\cdot)$ being the modular function defined by

$$
\rho_{\theta}(u)=\int_{\Omega} \theta(z, u(z)) d z=\int_{\Omega}\left(a(z)|u(z)|^{p}+|u(z)|^{q}\right) d z .
$$

We equip $L^{\theta}(\Omega)$ with the so-called "Luxemburg norm"

$$
\|u\|_{\theta}=\inf \left\{\lambda>0: \rho_{\theta}\left(\frac{u}{\lambda}\right) \leq 1\right\} .
$$

Then $L^{\theta}(\Omega)$ becomes a Banach space which is separable and reflexive (in fact, uniformly convex).

Using $L^{\theta}(\Omega)$ we can define the corresponding Musielak-Orlicz-Sobolev space $W^{1, \theta}(\Omega)$ as follows

$$
W^{1, \theta}(\Omega)=\left\{u \in L^{\theta}(\Omega):|D u| \in L^{\theta}(\Omega)\right\} .
$$

Here $D u$ denotes the weak gradient of $u(\cdot)$. We equip $W^{1, \theta}(\Omega)$ with the following norm

$$
\|u\|=\|u\|_{\theta}+\|D u\|_{\theta} \text { for all } u \in W^{1, \theta}(\Omega),
$$

where $\|D u\|_{\theta}=\||D u|\|_{\theta}$. This space is also a Banach space which is separable and reflexive (in fact, uniformly convex).

We have the following embeddings for these spaces (see Gasiński and Winkert [7]).

Proposition 1 If hypotheses $\mathrm{H}_{0}$ hold, then the following embeddings are true:

(a) $L^{\theta}(\Omega) \hookrightarrow L^{r}(\Omega)$ and $W^{1, \theta}(\Omega) \hookrightarrow W^{1, r}(\Omega)$ continuously for all $1 \leq r \leq q$;

(b) $W^{1, \theta}(\Omega) \hookrightarrow L^{r}(\Omega)$ continuously if $1 \leq r \leq q$ and compactly if $1 \leq r<q$;

(c) $L^{p}(\Omega) \hookrightarrow L^{\theta}(\Omega)$ continuously.

There is a close relation between the norm $\|\cdot\|_{\theta}$ and the modular function $\rho_{\theta}(\cdot)$. 
Proposition 2 If hypotheses $\mathrm{H}_{0}$ hold, then

(a) if $u \in L^{\theta}(\Omega) \backslash\{0\}$, then $\|u\|_{\theta}=\mu \Longleftrightarrow \rho_{\theta}\left(\frac{u}{\mu}\right)=1$;

(b) $\|u\|_{\theta}<1($ resp. $=1,>1) \Longleftrightarrow \rho_{\theta}(u)<1($ resp. $=1,>1)$;

(c) $\|u\|_{\theta}<1 \Longrightarrow\|u\|_{\theta}^{p} \leq \rho_{\theta}(u) \leq\|u\|_{\theta}^{q}$;

(d) $\|u\|_{\theta}>1 \Longrightarrow\|u\|_{\theta}^{q} \leq \rho_{\theta}(u) \leq\|u\|_{\theta}^{p}$;

(e) $\left\|u_{n}\right\|_{\theta} \rightarrow 0 \Longleftrightarrow \rho_{\theta}\left(u_{n}\right) \rightarrow 0$;

(f) $\left\|u_{n}\right\|_{\theta} \rightarrow+\infty \Longleftrightarrow \rho_{\theta}\left(u_{n}\right) \rightarrow+\infty$.

Let $\langle\cdot, \cdot\rangle$ denote the duality brackets for the pair $\left\langle W^{1, \theta}(\Omega)^{*}, W^{1, \theta}(\Omega)\right\rangle$ and let $V$ : $W^{1, \theta}(\Omega) \mapsto W^{1, \theta}(\Omega)^{*}$ be the nonlinear operator defined by

$$
\langle V(u), h\rangle=\int_{\Omega} a(z)|D u|^{p-2}(D u, D h)_{\mathbb{R}^{N}} d z+\int_{\Omega}|D u|^{q-2}(D u, D h)_{\mathbb{R}^{N}} d z
$$

for all $u, h \in W^{1, \theta}(\Omega)$.

The next proposition summarizes the main properties of this nonlinear operator (see Liu and Dai [11]).

Proposition 3 If hypotheses $\mathrm{H}_{0}$ hold, then the operator $V: W^{1, \theta}(\Omega) \mapsto W^{1, \theta}(\Omega)^{*}$ is bounded (that is, it maps bounded sets to bounded sets), continuous, monotone and of type $(S)_{+}$, namely it has the following property:

$$
\text { " } u_{n} \stackrel{w}{\rightarrow} u \text { in } W^{1, \theta}(\Omega), \limsup _{n \rightarrow \infty}\left\langle V\left(u_{n}\right), u_{n}-u\right\rangle \leq 0
$$

imply that

$$
u_{n} \rightarrow u \text { in } W^{1, \theta}(\Omega) .
$$

For every $u \in W^{1, \theta}(\Omega)$, we set

$$
|u|=\|D u\|_{\theta}+\left(\int_{\Omega} \xi(z)|u|^{q} d z\right)^{1 / q} .
$$

Evidently, this is a norm on $W^{1, \theta}(\Omega)$.

Proposition 4 If hypotheses $\mathrm{H}_{0}$ hold, then $\|\cdot\|$ and $|\cdot|$ are equivalent norms on $W^{1, \theta}(\Omega)$.

Proof Since $\xi \in L^{\infty}(\Omega)$ (see hypotheses $\mathrm{H}_{0}$ ), we have

$$
\int_{\Omega} \xi(z)|u|^{q} d z \leq\|\xi\|_{\infty}\|u\|_{q}^{q} \leq c_{1}\|u\|_{q}^{q}
$$

for some $c_{1}>0$ (see Proposition 1).

Therefore

$$
|u|=\|D u\|_{\theta}+c_{1}^{1 / q}\|u\|_{\theta} \leq c_{2}\|u\|
$$

for some $c_{2}>0$, all $u \in W^{1, \theta}(\Omega)$.

Claim: There exists $c_{3}>0$ such that $c_{3}\|u\| \leq|u|$ for all $u \in W^{1, \theta}(\Omega)$.

Arguing by contradiction, suppose we can find $\left\{u_{n}\right\}_{n \in \mathbb{N}} \subseteq W^{1, \theta}(\Omega)$ such that

$$
\left|u_{n}\right|<\frac{1}{n}\left\|u_{n}\right\| \text { for all } n \in \mathbb{N} .
$$


Let $v_{n}=\frac{u_{n}}{\left\|u_{n}\right\|}(n \in \mathbb{N})$. Then we have

$$
\left\|v_{n}\right\|=1 \text { and }\left\|D v_{n}\right\|_{\theta}+\left(\int_{\Omega} \xi(z)\left|v_{n}\right|^{q} d z\right)^{1 / q}<\frac{1}{n} \text { for all } n \in \mathbb{N} .
$$

By passing to a subsequence if necessary, we may assume that

$$
v_{n} \stackrel{w}{\rightarrow} v \text { in } W^{1, \theta}(\Omega) \text { and } v_{n} \rightarrow v \text { in } L^{q}(\Omega) \text { (see Proposition } 1 \text { ). }
$$

Then from (3) and (4), we have

$$
\begin{aligned}
& \|D v\|_{\theta}+\left(\int_{\Omega} \xi(z)|v|^{q} d z\right)^{1 / q} \leq 0, \\
\Rightarrow & \|D v\|_{\theta}+\left(\int_{\Omega} \xi(z)|v|^{q} d z\right)^{1 / q}=0, \\
\Rightarrow & \|D v\|_{\theta}=0 \text { and so } v=\eta \in \mathbb{R} .
\end{aligned}
$$

From (5) we have

$$
|\eta|^{q} \int_{\Omega} \xi(z) d z=0
$$

Since $\int_{\Omega} \xi(z) d z>0$ (see hypotheses $\mathrm{H}_{0}$ ), we have that $\eta=0$ and so $v=0$. From (3) we have

$$
\begin{aligned}
& \left\|D v_{n}\right\|_{\theta} \rightarrow 0, \\
\Rightarrow & v_{n} \rightarrow 0 \text { in } W^{1, \theta}(\Omega),
\end{aligned}
$$

which contradicts the fact that $\left\|v_{n}\right\|=1$ for all $n \in \mathbb{N}$ (see (3)). Finally, (2) and the Claim imply that

$$
\|\cdot\| \text { and }|\cdot| \text { are equivalent norms on } W^{1, \theta}(\Omega) .
$$

This proof is now complete.

In a similar fashion we can show also the following result. In what follows, $\|\cdot\|_{1, q}$ denotes the norm of the Sobolev space $W^{1, q}(\Omega)$.

Proposition 5 If $\xi \in L^{\infty}(\Omega) \backslash\{0\}$ and $\xi(z) \geq 0$ for a.a. $z \in \Omega$, then $\|\cdot\|_{1, q}$ and $[u]=$ $\|D u\|_{q}+\left(\int_{\Omega} \xi(z)|u|^{q} d z\right)^{1 / q}$ are equivalent norms on $W^{1, q}(\Omega)$.

Given $u \in W^{1, \theta}(\Omega)$, we define

$$
u^{+}(z)=\max \{u(z), 0\}, u^{-}(z)=\max \{-u(z), 0\} \text { for all } z \in \Omega .
$$

We know that

$$
u^{ \pm} \in W^{1, \theta}(\Omega), u=u^{+}-u^{-},|u|=u^{+}+u^{-} .
$$

If $X$ is a Banach space and $\varphi \in C^{1}(X, \mathbb{R})$, then

$$
K_{\varphi}=\left\{u \in X: \varphi^{\prime}(u)=0\right\} \text { (the critical set of } \varphi \text { ). }
$$


Also, if $c \in \mathbb{R}$, then

$$
\varphi^{c}=\{u \in X: \varphi(u) \leq c\} .
$$

Now we introduce the hypotheses on the reaction $f(z, x)$.

$\mathrm{H}_{1}: f: \Omega \times \mathbb{R} \mapsto \mathbb{R}$ is a Carathéodory function such that $f(z, 0)=0$ for a.a. $z \in \Omega$ and

(i) $|f(z, x)| \leq \hat{a}(z)\left(1+|x|^{r-1}\right)$ for a.a. $z \in \Omega$, all $x \in \mathbb{R}$ with $\hat{a} \in L^{\infty}(\Omega)$ and $p<r<q^{*}$;

(ii) if $F(z, x)=\int_{0}^{x} f(z, s) d s$, then $\lim _{x \rightarrow \pm \infty} \frac{F(z, x)}{|x|^{p}}=+\infty$ uniformly for a.a. $z \in \Omega$;

(iii) for a.a. $z \in \Omega$, the quotient function $x \mapsto \frac{f(z, x)}{|x|^{p-1}}$ is increasing on $\mathbb{R} \backslash\{0\}$;

(iv) $\lim _{x \rightarrow 0} \frac{f(z, x)}{|x|^{q-2} x}=0$ uniformly for a.a. $z \in \Omega$.

Remark 2 For $s \geq 0$ and $x \neq 0$, we have

$$
\begin{aligned}
& \frac{1-s^{p}}{p} f(z, x) x+F(z, s x)-F(z, x) \\
= & \int_{s}^{1} f(z, x) x t^{p-1} d t-\int_{s}^{1} \frac{d}{d t} F(z, t x) d t \\
= & \int_{s}^{1} f(z, x) x t^{p-1} d t-\int_{s}^{1} f(z, t x) x d t \text { (by the chain rule) } \\
= & \int_{s}^{1}\left(\frac{f(z, x)}{|x|^{p-1}}-\frac{f(z, t x)}{t^{p-1}|x|^{p-1}}\right)|x|^{p-1} x t^{p-1} d t \\
\geq & 0\left(\text { see hypothesis } \mathrm{H}_{1}(\mathrm{iii})\right), \\
\Rightarrow & F(z, x)-F(z, s x) \leq \frac{1-s^{p}}{p} f(z, x) x \\
& \quad \text { for a.a. } z \in \Omega, \text { all } x \in \mathbb{R}, \text { all } s \geq 0 .
\end{aligned}
$$

If in (6) we choose $s=0$, then

$$
\begin{aligned}
& p F(z, x) \leq f(z, x) x \text { for a.a. } z \in \Omega, \text { all } x \in \mathbb{R}, \\
\Rightarrow & \lim _{x \rightarrow \pm \infty} \frac{f(z, x)}{|x|^{p-2} x}=+\infty \text { uniformly for a.a. } z \in \Omega\left(\text { see hypothesis } \mathrm{H}_{1}(\mathrm{ii})\right) .
\end{aligned}
$$

So, we see that $f(z, \cdot)$ is $(p-1)$-superlinear as $x \rightarrow \pm \infty$. However this superlinearity property is not formulated using the AR-condition which is common in superlinear problems, see Liu \& Dai [11], hypothesis $\left(f_{4}\right)$. Also, in hypothesis $\mathrm{H}_{1}$ (iii) we do not require that the quotient function $x \mapsto \frac{f(z, x)}{|x|^{p-1}}$ is strictly increasing on $\mathbb{R} \backslash\{0\}$ as it is common in the literature, see Liu \& Dai [11], hypothesis $\left(f_{6}\right)$. Compared to the other superlinear Dirichlet double phase work of Gasiński \& Papageorgiou [5], we do not require $f(z, \cdot)$ to be locally Lipschitz and replace hypothesis $\mathrm{H}(f)$ (iii) of [5] by hypothesis $\mathrm{H}_{1}(\mathrm{iii})$. In case $f(z, \cdot)$ is strictly differentiable, then hypothesis $\mathrm{H}(f)$ (iii) of [5] has the form $(p-1) f(z, x) x \leq f_{x}^{\prime}(z, x) x^{2}$ for a.a. $z \in \Omega$, all $x \in \mathbb{R}$ (see Clarkc [4,p. 33]), which of course implies the monotonicity of the quotient function. Note that hypothesis $\mathrm{H}_{1}$ (iii) is equivalent to saying that for a.a. $z \in \Omega$, the mapping $x \mapsto \frac{f(z, x) x}{|x|^{p}}$ is increasing in $|x| \neq 0$.

First we will study problem (1) under the following stronger set of hypotheses. 
$\mathrm{H}_{1}^{\prime}: f: \Omega \times \mathbb{R} \mapsto \mathbb{R}$ is a Carathéodory function such that $f(z, 0)=0$ for a.a. $z \in \Omega$, hypotheses $\mathrm{H}_{1}^{\prime}(\mathrm{i})$, (ii), (iv) are the same as the corresponding hypotheses $\mathrm{H}_{1}$ (i), (ii), (iv) and (iii) for a.a. $z \in \Omega$, the quotient function $x \mapsto \frac{f(z, x)}{|x|^{p-1}}$ is strictly increasing on $\mathbb{R} \backslash\{0\}$.

Example 1 The following function satisfies hypotheses $\mathrm{H}_{1}$ but not $\mathrm{H}_{1}^{\prime}$. For the sake of simplicity we drop the $z$-dependence.

$$
f(x)= \begin{cases}|x|^{p-2} x & \text { if }|x| \leq 1, \\ |x|^{p-2} x(\ln |x|+1) & \text { if } 1<|x| .\end{cases}
$$

This function does not satisfy the AR-condition and so it does not fit in the framework of Liu \& Dai [11]. Also it does not fit the framework of Gasiński \& Papageorgiou [5] since it does not satisfy hypothesis $\mathrm{H}(f)$ (iii) of [5].

In what follows, $\rho_{a}(\cdot)$ denotes the modular function defined by

$$
\rho_{a}(D u)=\int_{\Omega} a(z)|D u|^{p} d z \text { for all } u \in W^{1, \theta}(\Omega) .
$$

Let $\varphi: W^{1, \theta}(\Omega) \mapsto \mathbb{R}$ be the energy (Euler) functional for problem (1) defined by

$$
\varphi(u)=\frac{1}{p} \rho_{a}(D u)+\frac{1}{q}\|D u\|_{q}^{q}+\frac{1}{q} \int_{\Omega} \xi(z)|u|^{q} d z-\int_{\Omega} F(z, u) d z
$$

for all $u \in W^{1, \theta}(\Omega)$.

We have that $\varphi \in C^{1}\left(W^{1, \theta}(\Omega)\right)$ and the Nehari manifold $\mathrm{N}$ of $\varphi(\cdot)$, is defined by

$$
\mathrm{N}=\left\{u \in W^{1, \theta}(\Omega):\left\langle\varphi^{\prime}(u), u\right\rangle=0, u \neq 0\right\} .
$$

Evidently, every weak solution of problem (1) is in the Nehari manifold. Since we want to produce constant sign solution for problem (1), we introduce the $C^{1}$-functionals $\varphi_{ \pm}$: $W^{1, \theta}(\Omega) \mapsto \mathbb{R}$ defined by

$$
\varphi_{ \pm}(u)=\frac{1}{p} \rho_{a}(D u)+\frac{1}{q}\|D u\|_{q}^{q}+\frac{1}{q} \int_{\Omega} \xi(z)|u|^{q} d z-\int_{\Omega} F\left(z, \pm u^{ \pm}\right) d z .
$$

We associate with these functionals, the following submanifolds of $\mathrm{N}$

$$
\begin{aligned}
& \mathrm{N}_{+}=\left\{u \in W^{1, \theta}(\Omega):\left\langle\varphi_{+}^{\prime}(u), u\right\rangle=0, u \geq 0, u \neq 0\right\}, \\
& \mathrm{N}_{-}=\left\{u \in W^{1, \theta}(\Omega):\left\langle\varphi_{-}^{\prime}(u), u\right\rangle=0, u \leq 0, u \neq 0\right\} .
\end{aligned}
$$

Finally, in order to produce a nodal (sign changing) solution of problem (1), we introduce the set

$$
\mathrm{N}_{0}=\left\{u \in W^{1, \theta}(\Omega):\left\langle\varphi^{\prime}(u), u^{+}\right\rangle=\left\langle\varphi^{\prime}(u), u^{-}\right\rangle=0, u^{ \pm} \neq 0\right\} .
$$

Note that

$$
\mathrm{N}_{+}, \mathrm{N}_{-}, \mathrm{N}_{0} \subseteq \mathrm{N} \text {. }
$$

For the study of $\mathrm{N}$ and of its submanifolds, we introduce the fibering function. So, let $u \in W^{1, \theta}(\Omega) \backslash\{0\}$ and consider the function

$$
k_{u}(t)=\varphi(t u) \text { for all } t \geq 0 .
$$

This is the fibering function for $u$. Note that

$$
t u \in \mathrm{N} \text { if and only if } k_{u}^{\prime}(t)=0 .
$$




\section{Constant sign solutions under strict monotonicity}

In this section, working with the functionals $\varphi_{ \pm}$and the submanifolds $\mathrm{N}_{ \pm}$, we will produce two constant sign (positive and negative) solutions for problem (1) under $\mathrm{H}_{1}^{\prime}$.

The Nehari manifold $\mathrm{N}$ is much smaller than the space $W^{1, \theta}(\Omega)$. So, the energy functional $\varphi(\cdot)$ restricted on $\mathrm{N}$ may exhibit properties which fail globally. In our case note that on account of hypothesis $\mathrm{H}_{1}(\mathrm{ii}), \varphi(\cdot)$ is unbounded below. However, as we show in the next proposition, $\left.\varphi\right|_{\mathrm{N}}$ is coercive, thus bounded below.

Proposition 6 If hypotheses $\mathrm{H}_{0}, \mathrm{H}_{1}$ hold, then $\left.\varphi\right|_{\mathrm{N}}$ is coercive.

Proof Arguing by contradiction, suppose we can find $\left\{u_{n}\right\}_{n \in \mathbb{N}} \subseteq W^{1, \theta}(\Omega)$ such that $u_{n} \in \mathrm{N}$ for all $n \in \mathbb{N},\left\|u_{n}\right\| \rightarrow+\infty, \varphi\left(u_{n}\right) \leq c_{4}$ for some $c_{4}>0$, all $n \in \mathbb{N}$.

Since $u_{n} \in \mathrm{N}$, we have

$$
\begin{aligned}
\rho_{a}\left(D u_{n}\right)+\left\|D u_{n}\right\|_{q}^{q}+\int_{\Omega} \xi(z)|u|^{q} d z & =\int_{\Omega} f\left(z, u_{n}\right) u_{n} d z, \\
\Rightarrow \frac{1}{p} \rho_{a}\left(D u_{n}\right)+\frac{1}{p}\left\|D u_{n}\right\|_{q}^{q}+\frac{1}{p} \int_{\Omega} \xi(z)\left|u_{n}\right|^{q} d z & =\frac{1}{p} \int_{\Omega} f\left(z, u_{n}\right) u_{n} d z \\
& \geq \int_{\Omega} F\left(z, u_{n}\right) d z \\
& \text { for all } n \in \mathbb{N}(\text { see (7)). }
\end{aligned}
$$

From (9) and (10), we obtain

$$
\left(\frac{1}{q}-\frac{1}{p}\right)\left(\left\|D u_{n}\right\|_{q}^{q}+\int_{\Omega} \xi(z)\left|u_{n}\right|^{q} d z\right) \leq c_{4} \text { for all } n \in \mathbb{N},
$$

$\Rightarrow\left\{u_{n}\right\}_{n \in \mathbb{N}} \subseteq W^{1, q}(\Omega)$ is bounded (see Proposition 5),

$\Rightarrow\left\{u_{n}\right\}_{n \in \mathbb{N}} \subseteq L^{r}(\Omega)$ is relatively compact (see Proposition 1 ).

From (9), (11) and (12) it follows that

$$
\begin{aligned}
& \frac{1}{p} \rho_{a}\left(D u_{n}\right) \leq c_{5} \text { for some } c_{5}>0, \text { all } n \in \mathbb{N}, \\
\Rightarrow & \rho_{\theta}\left(D u_{n}\right)+\int_{\Omega} \xi(z)\left|u_{n}\right|^{q} d z \leq c_{6} \text { for some } c_{6}>0, \text { all } n \in \mathbb{N}(\text { see (11), (13)), } \\
\Rightarrow & \left\{u_{n}\right\}_{n \in \mathbb{N}} \subseteq W^{1, \theta}(\Omega) \text { is bounded (see Proposition 4). }
\end{aligned}
$$

Comparing (9) and (14), we have a contradiction. This proves that $\left.\varphi\right|_{\mathrm{N}}$ is coercive.

Clearly we have

$$
\left.\varphi_{+}\right|_{\mathrm{N}_{+}}=\left.\varphi\right|_{\mathrm{N}_{+}},\left.\varphi\right|_{-} \mathrm{N}_{-}=\left.\varphi\right|_{\mathrm{N}_{-}} .
$$

Since $\mathrm{N}_{+}, \mathrm{N}_{-} \subseteq \mathrm{N}$ (see (8)), from Proposition 6 we infer the following corollary.

Corollary 7 If hypotheses $\mathrm{H}_{0}, \mathrm{H}_{1}$ hold, then $\left.\varphi_{+}\right|_{\mathrm{N}_{+}}$and $\left.\varphi_{-}\right|_{\mathrm{N}_{-}}$are both coercive.

Next we show that $\mathrm{N} \neq \emptyset$. Now we will start using the stronger hypotheses $\mathrm{H}_{1}^{\prime}$. 
Proposition 8 If hypotheses $\mathrm{H}_{0}, \mathrm{H}_{1}^{\prime}$ hold and $u \in W^{1, \theta}(\Omega) \backslash\{0\}$, then there exists a unique $t_{u}>0$ such that

$$
t_{u} u \in \mathrm{N} \text {. }
$$

Proof We introduce the function $\beta_{u}: \mathbb{R}_{+} \mapsto \mathbb{R}$ defined by

$$
\beta_{u}(t)=t^{p} \rho_{a}(D u)+t^{q}\left(\|D u\|_{q}^{q}+\int_{\Omega} \xi(z)|u|^{q} d z\right)-\int_{\Omega} f(z, t u)(t u) d z
$$

for all $t \geq 0$.

We see that

$$
\beta_{u}(t)=t k_{u}^{\prime}(t) \text { for all } t \geq 0 .
$$

Hypothesis $\mathrm{H}_{1}^{\prime}$ (iii) implies that if $x \neq 0$ and $t \in(0,1)$, then

$$
\begin{aligned}
& \frac{f(z, t x)(t x)}{t^{p}|x|^{p}}<\frac{f(z, x) x}{|x|^{p}} \text { for a.a. } z \in \Omega, \\
\Rightarrow & f(z, t x)(t x)<t^{p} f(z, x) x \text { for a.a. } z \in \Omega, \text { all } x \neq 0, \text { all } t \in(0,1) .
\end{aligned}
$$

We use (17) in (15) and obtain

$$
\begin{aligned}
\beta_{u}(t) & \geq t^{p} \rho_{a}(D u)+t^{q}\left(\|D u\|_{q}^{q}+\int_{\Omega} \xi(z)|u|^{q} d z\right)-t^{p} \int_{\Omega} f(z, u) u d z \\
& \geq t^{q} \frac{1}{2^{q-1}}[u]^{q}-t^{p} \int_{\Omega} f(z, u) u d z .
\end{aligned}
$$

Since $q<p$, we see that

$$
\beta_{u}(t)>0 \text { for all } t \in(0,1) \text { small. }
$$

Also we have

$$
\begin{aligned}
& \frac{\beta_{u}(t)}{t^{p}}=\rho_{a}(D u)+\frac{1}{t^{p-q}}\left(\|D u\|_{q}^{q}+\int_{\Omega} \xi(z)|u|^{q} d z\right)-\int_{\Omega} \frac{f(z, t u)(t u)}{t^{p}} d z \\
& \leq \rho_{a}(D u)+\frac{2}{t^{p-q}}[u]^{q}-\int_{\Omega} \frac{p F(z, t u)}{t^{p}} d z(\text { see }(7)) \\
& \Rightarrow\left.\lim _{t \rightarrow+\infty} \frac{\beta_{u}(t)}{t^{p}}=-\infty \text { (see hypothesis } \mathrm{H}_{1}^{\prime}(\mathrm{ii})\right), \\
& \Rightarrow \beta_{u}(t)<0 \text { for } t>0 \text { large. }
\end{aligned}
$$

Then relations (18), (19) and Bolzano's theorem, imply that there exists $t_{u}>0$ such that

$$
\begin{aligned}
& \beta_{u}\left(t_{u}\right)=0, \\
\Rightarrow & t_{u} k_{u}^{\prime}\left(t_{u}\right)=0(\text { see }(16)), \\
\Rightarrow & k_{u}^{\prime}\left(t_{u}\right)=0, \\
\Rightarrow & t_{u} u \in \mathrm{N} .
\end{aligned}
$$

Finally, note that

$$
\beta_{u}(t)=0 \Longleftrightarrow \rho_{u}(D u)=\int_{\Omega} \frac{f(z, t u)(t u)}{t^{p}} d z-\frac{1}{t^{p-q}}\left(\|D u\|_{q}^{q}+\int_{\Omega} \xi(z)|u|^{q} d z\right) .
$$

In the last equality the right-hand side is strictly increasing in $t>0$ (see hypothesis $\mathrm{H}_{1}^{\prime}$ (ii)) and so it follows that $t_{u}>0$ is unique. 
In a similar fashion, working with $\varphi_{+}$and $\varphi_{-}$, we show the nonemptiness of the submanifolds $\mathrm{N}_{+}$and $\mathrm{N}_{-}$, respectively. Recall that $\mathrm{N}_{ \pm} \subseteq \mathrm{N}$ (see (8)).

Proposition 9 If hypotheses $\mathrm{H}_{0}, \mathrm{H}_{1}^{\prime}$ hold and $u \in W^{1, \theta}(\Omega) \backslash\{0\}$ with $u \geq 0$ (resp. $\left.u \leq 0\right)$, then there exists a unique $t_{u}^{+}>0$ (resp. $\left.t_{u}^{-}>0\right)$ such that

$$
t_{u}^{+} u \in \mathrm{N}_{+}\left(\text {resp. } t_{u}^{-} u \in \mathrm{N}_{-}\right) \text {. }
$$

The next proposition presents a useful connection between the fibering function and the elements of the Nehari manifold.

Proposition 10 If hypotheses $\mathrm{H}_{0}, \mathrm{H}_{1}^{\prime}$ hold and $u \in \mathrm{N}$, then $\varphi(t u) \leq \varphi(u)$ for all $t \geq 0$.

Proof From (16) we have

$$
\begin{aligned}
\beta_{u}(1) & =k_{u}^{\prime}(1), \\
\Rightarrow \quad \beta_{u}(1) & =0(\text { since } u \in \mathrm{N}) .
\end{aligned}
$$

Note that

$$
\begin{aligned}
\frac{k_{u}(t)}{t^{p}} & =\frac{1}{p} \rho_{a}(D u)+\frac{1}{q t^{p-q}}\left(\|D u\|_{q}^{q}+\int_{\Omega} \xi(z)|u|^{q} d z\right)-\int_{\Omega} \frac{F(z, t u)}{t^{p}} d z, \\
\Rightarrow \frac{k_{u}(t)}{t^{p}} & \left.\rightarrow-\infty \text { as } t \rightarrow+\infty \text { (see hypothesis } H_{1}^{\prime}(i i)\right), \\
\Rightarrow k_{u}(t) & <0 \text { for all } t>0 \text { large. }
\end{aligned}
$$

On the other hand, on account of hypotheses $\mathrm{H}_{1}^{\prime}$ (i), (iv) given $\varepsilon>0$, we can find $c_{7}=$ $c_{7}(\varepsilon)>0$ such that

$$
F(z, x) \leq \frac{\varepsilon}{q}|x|^{q}+c_{7}|x|^{r} \text { for a.a. } z \in \Omega, \text { all } x \in \mathbb{R} .
$$

Then using (21) we have

$$
k_{u}(t) \geq \frac{t^{p}}{p} \rho_{a}(D u)+\frac{t^{q}}{q}\left(\frac{1}{2^{q-1}}-\varepsilon c_{8}\right)[u]^{q}-t^{r} c_{7}\|u\|_{r}^{r}
$$

for some $c_{8}>0$ (see Proposition 5).

Choosing $\varepsilon \in\left(0, \frac{1}{2^{q-1} c_{8}}\right)$, we obtain

$$
\begin{aligned}
k_{u}(t) & \geq c_{9} t^{p}-c_{10} t^{r} \text { for some } c_{9}, c_{10}>0, \text { all } t \in(0,1), \\
\Rightarrow & \left.k_{u}(t)>0 \text { for all } t \in(0,1) \text { small (recall that } p<r\right) .
\end{aligned}
$$

From (21), (22) it follows that there exists $\hat{t}_{u}>0$ local maximizer of the fibering function. Then

$$
\begin{aligned}
& k_{u}^{\prime}\left(\hat{t}_{u}\right)=0, \\
\Rightarrow & \hat{t}_{u} u \in \mathrm{N}, \\
\Rightarrow & \hat{t}_{u}=1 \text { and it is a global maximizer of } k_{u}(\cdot) \\
& (\text { recall that } u \in \mathrm{N} \text { and see Proposition } 8), \\
\Rightarrow & \varphi(t u) \leq \varphi(u) \text { for all } t \geq 0 .
\end{aligned}
$$

This proof is now complete. 
The same result is also true for $\varphi_{ \pm}$.

Proposition 11 If hypotheses $\mathrm{H}_{0}, \mathrm{H}_{1}^{\prime}$ hold and $u \in \mathrm{N}_{+}$(resp. $\left.u \in \mathrm{N}_{-}\right)$, then $\varphi_{+}(t u) \leq \varphi_{+}(u)$ (resp. $\left.\varphi_{-}(t u) \leq \varphi_{-}(u)\right)$ for all $t \geq 0$.

In the next proposition we show that the elements of the Nehari manifold are in norm bounded away from zero.

Proposition 12 If hypotheses $\mathrm{H}_{0}, \mathrm{H}_{1}^{\prime}$ hold, then there exists $\mu_{0}>0$ such that

$$
0<\mu_{0} \leq\|u\|_{r},\|u\| \text { for all } u \in \mathrm{N} .
$$

Proof From hypotheses $\mathrm{H}_{1}^{\prime}(\mathrm{i})$, (iv), we see that given $\varepsilon>0$, we can find $c_{11}=c_{11}(\varepsilon)>0$ such that

$$
f(z, x) x \leq \varepsilon|x|^{q}+c_{11}|x|^{r} \text { for a.a. } z \in \Omega, \text { all } x \in \mathbb{R} .
$$

Let $u \in \mathrm{N}$ and we can always assume that $\|u\|,|u| \leq 1$. Then

$$
\begin{aligned}
& \rho_{a}(D u)+\left(\|D u\|_{q}^{q}+\int_{\Omega} \xi(z)|u|^{q} d z\right) \\
\leq & \varepsilon c_{12}[u]^{q}+c_{11}\|u\|_{r}^{r} \text { for some } c_{12}>0, \\
\Rightarrow & \rho_{a}(D u)+\left(\frac{1}{2^{q-1}}-\varepsilon c_{12}\right)[u]^{q} \leq c_{11}\|u\|_{r}^{r} .
\end{aligned}
$$

Choosing $\varepsilon \in\left(0, \frac{1}{2^{q-1} c_{12}}\right)$, we obtain

$$
\begin{aligned}
& \rho_{\theta}(D u)+\int_{\Omega} \xi(z)|u|^{q} d z \leq c_{13}\|u\|_{r}^{r} \text { for some } c_{13}>0, \\
\Rightarrow & \rho_{\theta}(D u)+\left(\int_{\Omega} \xi(z)|u|^{q} d z\right)^{p / q} \leq c_{14}\|u\|_{r}^{r} \\
& \text { for some } c_{14}>0(\text { recall that }|u| \leq 1 \text { and that } q<p), \\
\Rightarrow & \left.\|D u\|_{\theta}^{p}+\left(\int_{\Omega} \xi(z)|u|^{q} d z\right)^{p / q} \leq c_{14}\|u\|_{r}^{r} \text { (see Proposition } 2\right), \\
\Rightarrow & |u|^{p} \leq c_{15}\|u\|_{r}^{r} \text { for some } c_{15}>0, \\
\Rightarrow & \|u\|^{p} \leq c_{16}\|u\|_{r}^{r} \text { for some } c_{16}>0 .
\end{aligned}
$$

Therefore we conclude that we can find $\mu_{0}>0$ such that

$$
0<\mu_{0} \leq\|u\|_{r},\|u\| \text { for all } u \in \mathrm{N} .
$$

This proof is now complete.

From (8) it follows the following auxiliary property.

Corollary 13 If hypotheses $\mathrm{H}_{0}, \mathrm{H}_{1}^{\prime}$ hold, then

$$
0<\mu_{0} \leq\|u\|_{r},\|u\| \text { for all } u \in \mathrm{N}_{+} .
$$

Now let

$$
m_{+}=\inf _{\mathrm{N}_{+}} \varphi_{+} \text {and } m_{-}=\inf _{\mathrm{N}_{-}} \varphi_{-} .
$$

We show that both infima are attained. 
Proposition 14 If hypotheses $\mathrm{H}_{0}, \mathrm{H}_{1}^{\prime}$ hold, then there exist $u_{0} \in \mathrm{N}_{+}$and $v_{0} \in \mathrm{N}_{-}$such that

$$
\varphi_{+}\left(u_{0}\right)=m_{+} \text {and } \varphi_{-}\left(v_{0}\right)=m_{-} .
$$

Proof We consider a sequence $\left\{u_{n}\right\}_{n \in \mathbb{N}} \subseteq W^{1, \theta}(\Omega)$ such that

$$
u_{n} \in \mathrm{N}_{+} \text {for all } n \in \mathbb{N} \text { and } \varphi_{+}\left(u_{n}\right) \downarrow m_{+} .
$$

From Corollary 7, we know that $\left\{u_{n}\right\}_{n \in \mathbb{N}} \subseteq W^{1, \theta}(\Omega)$ is bounded and so by passing to a subsequence, we may assume that

$$
u_{n} \stackrel{w}{\rightarrow} u_{0} \text { in } W^{1, \theta}(\Omega) \text { and } u_{n} \rightarrow u_{0} \text { in } L^{r}(\Omega) .
$$

Since $\mathrm{N}_{+} \subseteq \mathrm{N}$ (see (8)), we have

$$
\rho_{a}\left(D u_{n}\right)+\left\|D u_{n}\right\|_{q}^{q}+\int_{\Omega} \xi(z)\left|u_{n}\right|^{q} d z=\int_{\Omega} f\left(z, u_{n}\right) u_{n} d z, u_{n} \geq 0 \text { for all } n \in \mathbb{N} .
$$

Then $u_{0} \geq 0$ (see (25)) and from Corollary 13 and (25), we see that $0<\mu_{0} \leq\left\|u_{0}\right\|_{r}$, hence $u_{0} \neq 0$.

From (25) and (26), we have

$$
\rho_{a}\left(D u_{0}\right)+\left\|D u_{0}\right\|_{q}^{q}+\int_{\Omega} \xi(z)\left|u_{0}\right|^{q} d z \leq \int_{\Omega} f\left(z, u_{0}\right) u_{0} d z .
$$

Suppose that the above inequality is strict, that is,

$$
\rho_{a}\left(D u_{0}\right)+\left\|D u_{0}\right\|_{q}^{q}+\int_{\Omega} \xi(z)\left|u_{0}\right|^{q} d z<\int_{\Omega} f\left(z, u_{0}\right) u_{0} d z .
$$

We use the function $\beta_{u_{0}}(\cdot)$ from the proof of Proposition 8 . We know that

$$
\beta_{u_{0}}(t)>0 \text { for } t \in(0,1) \text { small (see (18)), } \beta_{u_{0}}(1)<0 \text { (see (15), (27)). }
$$

So, we can find $t_{0} \in(0,1)$ such that

$$
\begin{aligned}
& \beta_{u_{0}}\left(t_{0}\right)=0, \\
\Rightarrow & t_{0} k_{u_{0}}^{\prime}\left(t_{0}\right)=0 \text { since } u_{0} \geq 0 \text { and so } \varphi_{+}\left(t u_{0}\right)=\varphi\left(t u_{0}\right), t \geq 0, \\
\Rightarrow & t_{0} u_{0} \in \mathrm{N}_{+} .
\end{aligned}
$$

We have

$$
\begin{aligned}
m_{+} \leq & \varphi_{+}\left(t_{0} u_{0}\right) \\
= & \frac{1}{p} \rho_{a}\left(D\left(t_{0} u_{o}\right)\right)+\frac{1}{q}\left(\left\|D\left(t_{0} u_{0}\right)\right\|_{q}^{q}+\int_{\Omega} \xi(z)\left|t_{0} u_{0}\right|^{q} d z\right)-\int_{\Omega} F\left(z, t_{0} u_{0}\right) d z \\
= & \frac{1}{p} \int_{\Omega}\left(f\left(z, t_{0} u_{0}\right)\left(t_{0} u_{0}\right)-p F\left(z, t_{0} u_{0}\right)\right) d z \\
& +\left(\frac{1}{q}-\frac{1}{p}\right)\left(\| D\left(t_{0} u_{0} \|_{q}^{q}+\int_{\Omega} \xi(z)\left|t_{0} u_{0}\right|^{q} d z\right)\right)\left(\text { since } t_{0} u_{0} \in \mathrm{N}_{+}\right) .
\end{aligned}
$$

On account of (6), we have

$$
f(z, s x)(s x)-p F(z, s x) \leq f(z, x) x-p F(z, x)
$$

for a.a. $z \in \Omega$, all $x \in \mathbb{R}$, all $s \in(0,1)$. 
Recall that $0<t_{0}<1$. So, using (27) and (29) in (28), we obtain

$$
\begin{aligned}
m_{+}< & \frac{1}{p} \int_{\Omega}\left(f\left(z, u_{0}\right) u_{0}-p F\left(z, u_{0}\right)\right) d z \\
& +\left(\frac{1}{q}-\frac{1}{p}\right)\left(\left\|D u_{0}\right\|_{q}^{q}+\int_{\Omega} \xi(z)\left|u_{0}\right|^{q} d z\right) \\
\leq & \liminf _{n \rightarrow \infty}\left(\frac{1}{p} \int_{\Omega}\left(f\left(z, u_{n}\right) u_{n}-p F\left(z, u_{n}\right)\right) d z\right. \\
& \left.+\left(\frac{1}{q}-\frac{1}{p}\right)\left(\left\|D u_{n}\right\|_{q}^{q}+\int_{\Omega} \xi(z)\left|u_{n}\right|^{q} d z\right)\right)(\operatorname{see}(25)) \\
= & \liminf _{n \rightarrow \infty}\left(\frac{1}{p} \rho_{a}\left(D u_{n}\right)+\frac{1}{q}\left(\left\|D u_{n}\right\|_{q}^{q}+\int_{\Omega} \xi(z)\left|u_{n}\right|^{q} d z\right)-\int_{\Omega} F\left(z, u_{n}\right) d z\right) \\
= & \liminf _{n \rightarrow \infty} \varphi_{+}\left(u_{n}\right)=m_{+},
\end{aligned}
$$

a contradiction.

So, we have

$$
\begin{aligned}
& \rho_{a}\left(D u_{0}\right)+\left\|D u_{0}\right\|_{q}^{q}+\int_{\Omega} \xi(z)\left|u_{0}\right|^{q} d z=\int_{\Omega} f\left(z, u_{0}\right) u_{0} d z, \\
\Rightarrow & u_{0} \in \mathrm{N}_{+} .
\end{aligned}
$$

Then it follows that

$$
\rho_{\theta}\left(D u_{n}\right) \rightarrow \rho_{\theta}\left(D u_{0}\right)(\text { see }(25)) .
$$

The uniform convexity of $\theta(z, \cdot)$ implies that

$$
\begin{aligned}
& \rho_{\theta}\left(D u_{n}-D u_{0}\right) \rightarrow 0 \text { (see Harjuletho \& Hästö [9, p. 65] ), } \\
\Rightarrow & \left.D u_{n} \rightarrow D u_{0} \text { in } L^{\theta}\left(\Omega, \mathbb{R}^{N}\right) \text { (see Proposition } 2\right), \\
\Rightarrow & u_{n} \rightarrow u_{0} \text { in } W^{1, \theta}(\Omega)(\operatorname{see}(25) \text { and Proposition } 4) .
\end{aligned}
$$

So, finally we can say that

$$
\varphi_{+}\left(u_{0}\right)=m_{+}, u_{0} \in \mathrm{N}_{+} .
$$

Similarly, working with $\varphi_{-}(\cdot)$ and $\mathrm{N}_{-}$, we show that there exists $v_{0} \in W^{1, \theta}(\Omega)$ such that

$$
\varphi_{-}\left(v_{0}\right)=m_{-}, v_{0} \in \mathrm{N}_{-} .
$$

This proof is now complete.

Next, we show that the two minimizers produced in Proposition 14 are in fact critical points of the functionals $\varphi_{+}$and $\varphi_{-}$respectively. This means that $\mathrm{N}_{+}$and $\mathrm{N}_{-}$are natural constraints for $\varphi_{+}$and $\varphi_{-}$, respectively (see Papapgeorgiou et al. [19, p. 422]). Our proof is inspired by the works of Willem [26, p. 74] and Szulkin and Weth [25, p. 611].

Proposition 15 If hypothesis $\mathrm{H}_{0}, \mathrm{H}_{1}^{\prime}$ hold and $u_{0} \in \mathrm{N}_{+}$and $v_{0} \in \mathrm{N}_{-}$are the two minimizers from Proposition 14, then $u_{0} \in K_{\varphi_{+}}$and $v_{0} \in K_{\varphi_{-}}$.

Proof Let $\tau \in \stackrel{\circ}{\mathbb{R}}_{+} \backslash\{1\}\left(\AA_{\mathbb{R}}^{+}=(0,+\infty)\right)$. Since $u_{0} \in \mathrm{N}_{+}$from Proposition 11 we have

$$
\varphi_{+}\left(\tau u_{0}\right) \leq \varphi_{+}\left(u_{0}\right)=m_{+} .
$$


We argue indirectly. So, suppose that $u_{0} \notin K_{\varphi_{+}}$. Then

$$
\varphi_{+}^{\prime}\left(u_{0}\right) \neq 0 \text {. }
$$

We can find $\delta>0$ and $d>0$ such that

$$
\left\|u-u_{0}\right\| \leq 3 \delta \Rightarrow\left\|\varphi_{+}^{\prime}(u)\right\|_{*} \geq d .
$$

We consider the interval $I=\left(\frac{1}{2}, \frac{3}{2}\right)$. From (30) we have

$$
\hat{m}_{+}=\max _{\tau \in \partial I} \varphi_{+}\left(\tau u_{0}\right)<m_{+} .
$$

We can always choose $\delta>0$ even smaller if necessary in order to have $\frac{d \delta}{8} \leq \frac{m_{+}-\hat{m}_{+}}{4}$. Then we apply the quantitative deformation theorem of Willem [26, p. 38] with $\varepsilon=\frac{d \delta}{8}$ and $S=\bar{B}_{\delta}\left(u_{0}\right)=\left\{u \in W^{1, \theta}(\Omega):\left\|u-u_{0}\right\| \leq \delta\right\}$. So, we produce a deformation $h$ : $[0,1] \times W^{1, \theta}(\Omega) \mapsto W^{1, \theta}(\Omega)$ such that

- $h(1, u)=u$ if $\varphi_{+}(u) \notin\left[m_{+}-2 \varepsilon, m_{+}+2 \varepsilon\right] \cap \bar{B}_{2 \delta}\left(u_{0}\right)$;

- $h\left(1, \varphi_{+}^{m_{+}+\varepsilon} \cap \bar{B}_{\delta}\left(u_{0}\right)\right) \subseteq \varphi_{+}^{m_{+}-\varepsilon}$;

- $\varphi_{+}(h(1, u)) \leq \varphi_{+}(u)$ for all $u \in W^{1, \theta}(\Omega)$.

From these properties of the deformation and (31), we deduce that

$$
\max _{\tau \in \bar{I}} \varphi_{+}\left(h\left(1, \tau u_{0}\right)\right)<m_{+} .
$$

Claim: $h\left(1, I u_{0}\right) \cap \mathrm{N}_{+} \neq \emptyset$.

Let $\psi(\tau)=h\left(1, \tau u_{0}\right)$ and consider the following two functions

$$
\begin{aligned}
& \sigma_{0}(\tau)=\left\langle\varphi_{+}^{\prime}\left(\tau u_{0}\right), u_{0}\right\rangle, \\
& \sigma_{1}(\tau)=\frac{1}{\tau}\left\langle\varphi_{+}^{\prime}(\psi(\tau)), \psi(\tau)\right\rangle, \tau>0 .
\end{aligned}
$$

Recall that $u_{0} \in \mathrm{N}_{+}$. So, we have

$$
\begin{aligned}
& \left\langle\varphi_{+}^{\prime}\left(\tau u_{0}\right), u_{0}\right\rangle>0 \text { if } 0<\tau<1, \\
& \left\langle\varphi_{+}^{\prime}\left(\tau u_{0}\right), u_{0}\right\rangle<0 \text { if } 1<\tau \\
& \text { (see Propositions } 11 \text { and } 9 \text { ). }
\end{aligned}
$$

By $d_{B}(\cdot, \cdot, \cdot)$ we denote the Brouwer degree. Then from (33), (34) and Lloyd [13, see Illustration 1, pp. 19, 20], we have

$$
d_{B}\left(\sigma_{0}, I, 0\right)=-1 .
$$

From the properties of the deformation, we have

$$
\begin{aligned}
& \left.\sigma_{0}\right|_{\partial I}=\left.\sigma_{1}\right|_{\partial I}, \\
\Rightarrow & d_{B}\left(\sigma_{0}, I, 0\right)=d_{B}\left(\sigma_{1}, I, 0\right), \\
\Rightarrow & d_{B}\left(\sigma_{1}, I, 0\right) \neq 0(\text { see }(35)), \\
\Rightarrow & \sigma_{1}(\tau)=0 \text { for some } \tau \in I(\text { see }[9],[19]), \\
\Rightarrow & \psi(\tau)=h\left(1, \tau u_{0}\right) \in \mathrm{N}_{+} .
\end{aligned}
$$


This proves the Claim.

But then on account of the Claim, we have

$$
m_{+} \leq \max _{\tau \in \bar{I}} \varphi_{+}\left(h\left(1, \tau u_{0}\right)\right) .
$$

Comparing with (32) we have a contradiction. Therefore $u_{0} \in K_{\varphi_{+}}$.

Similarly we show that $v_{0} \in K_{\varphi_{-}}$.

Now we are ready to produce two constant sign solutions for problem (1) (positive and negative solutions), under the strict monotonicity condition $\mathrm{H}_{1}^{\prime}$ (iii).

Proposition 16 If hypotheses $\mathrm{H}_{0}, \mathrm{H}_{1}^{\prime}$ hold, then problem (1) has at least two nontrivial constant sign solutions

$$
\begin{aligned}
& u_{0} \in \mathrm{N}_{+} \cap L^{\infty}(\Omega), u_{0}(z)>0 \text { for a.a. } z \in \Omega, \\
& v_{0} \in \mathrm{N}_{-} \cap L^{\infty}(\Omega), v_{0}(z)<0 \text { for a.a. } z \in \Omega .
\end{aligned}
$$

Proof From Proposition 15 we know that

$$
u_{0} \in \mathrm{N}_{+} \text {and } v_{0} \in \mathrm{N}_{-}
$$

are nontrivial constant sign solutions of problem (1). As in Gasiński and Winkert [6, Theorem 3.1], we have that

$$
u_{0} \in \mathrm{N}_{+} \cap L^{\infty}(\Omega), v_{0} \in \mathrm{N}_{-} \cap L^{\infty}(\Omega)
$$

Finally using Proposition 2.4 of Papageorgiou, Vetro and Vetro [22], we have

$$
v_{0}(z)<0<u_{0}(z) \text { for a.a. } z \in \Omega \text {. }
$$

This proof is now complete.

\section{Nodal solutions under strict monotonicity}

In this section we prove the existence of a nodal (that is, sign changing) solution for problem (1), under the strict monotonicity hypothesis $\mathrm{H}_{1}^{\prime}$ (iii). For this purpose, we bring in the picture the submanifold $\mathrm{N}_{0}$.

We define

$$
m_{0}=\inf _{\mathrm{N}_{0}} \varphi
$$

Proposition 17 If hypotheses $\mathrm{H}_{0}, \mathrm{H}_{1}^{\prime}$ hold, then there exists $y_{0} \in \mathrm{N}_{0}$ such that

$$
m_{0}=\varphi\left(y_{0}\right) .
$$

Proof We consider a sequence $\left\{y_{n}\right\}_{n \in \mathbb{N}} \subseteq W^{1, \theta}(\Omega)$ such that

$$
y_{n} \in \mathrm{N}_{0} \text { for all } n \in \mathbb{N} \text { and } \varphi\left(y_{n}\right) \downarrow m_{0} .
$$

From Proposition 6 we know that $\left\{y_{n}\right\}_{n \in \mathbb{N}} \subseteq W^{1, \theta}(\Omega)$ is bounded. Then $\left\{y_{n}^{+}\right\}_{n \in \mathbb{N}} \subseteq$ $W^{1, \theta}(\Omega)$ and $\left\{y_{n}^{-}\right\}_{n \in \mathbb{N}} \subseteq W^{1, \theta}(\Omega)$ are bounded, too. So, we may assume that

$$
y_{n}^{+} \stackrel{w}{\rightarrow} \hat{y}_{1} \text { and } y_{n}^{-} \stackrel{w}{\rightarrow} \hat{y}_{2} \text { in } W^{1, \theta}(\Omega) .
$$


Suppose that $\hat{y}_{1}=0$. Since $y_{n} \in \mathrm{N}$, we have that $y_{n}^{+} \in \mathrm{N}_{+}$for all $n \in \mathbb{N}$ and so

$$
\begin{aligned}
\rho_{a}\left(D y_{n}^{+}\right)+\left\|D y_{n}^{+}\right\|_{q}^{q}+\int_{\Omega} \xi(z)\left(y_{n}^{+}\right)^{q} d z & =\int_{\Omega} f\left(z, y_{n}^{+}\right) y_{n}^{+} d z \text { for all } n \in \mathbb{N}, \\
\Rightarrow & \rho_{\theta}\left(D y_{n}^{+}\right)+\int_{\Omega} \xi(z)\left(y_{n}^{+}\right)^{q} d z \rightarrow 0 \\
& \left(\text { see }(36) \text { and recall that } \hat{y}_{1}=0\right), \\
\Rightarrow & \left|y_{n}^{+}\right| \rightarrow 0, \\
\Rightarrow & y_{n}^{+} \rightarrow 0 \text { in } W^{1, \theta}(\Omega) \text { (see Proposition 4). }
\end{aligned}
$$

But from Corollary 13 we know that

$$
0<\mu_{0} \leq\left\|y_{n}^{+}\right\| \text {for all } n \in \mathbb{N} .
$$

Comparing (37) and (38), we have a contradiction.

Therefore $\hat{y}_{1} \neq 0$. Similarly we show that $\hat{y}_{2} \neq 0$.

According to Proposition 9, there exist a unique $\hat{t}_{1}, \hat{t}_{2}>0$ such that

$$
\hat{t}_{1} \hat{y}_{1} \in \mathrm{N}_{+} \text {and } \hat{t}_{2} \hat{y}_{2} \in \mathrm{N}_{-} \text {. }
$$

We set

$$
y_{0}=\hat{t}_{1} \hat{y}_{1}-\hat{t}_{2} \hat{y}_{2}
$$

Evidently we have

$$
y_{0}^{+}=\hat{t}_{1} \hat{y}_{1}, y_{0}^{-}=\hat{t}_{2} \hat{y}_{2} \text { and so } y_{0} \in \mathrm{N}_{0} \text {. }
$$

We have

$$
\begin{aligned}
m_{0} & =\lim _{n \rightarrow \infty} \varphi\left(y_{n}\right) \\
& =\lim _{n \rightarrow \infty}\left(\varphi_{+}\left(y_{n}^{+}\right)+\varphi_{-}\left(-y_{n}^{-}\right)\right) \\
& \geq \liminf _{n \rightarrow \infty}\left(\varphi_{+}\left(\hat{t}_{1} y_{n}^{+}\right)+\varphi_{-}\left(-\hat{t}_{2} y_{n}^{-}\right)\right) \text {(see Proposition 11) } \\
& \geq \varphi\left(y_{0}\right) \\
& \geq m_{0}\left(\text { since } y_{0} \in \mathrm{N}_{0}\right), \\
\Rightarrow & m_{0}=\varphi\left(y_{0}\right) \text { with } y_{0} \in \mathrm{N}_{0} .
\end{aligned}
$$

This proof is now complete.

Reasoning as in the proof of Proposition 15, we show that $\mathrm{N}_{0}$ is a natural constraint for $\varphi(\cdot)$, that is, $y_{0} \in K_{\varphi}$. In this case we apply the quantitative deformation theorem of Willem [26], using the following data: the set

$$
D=\left(\frac{1}{2}, \frac{3}{2}\right) \times\left(\frac{1}{2}, \frac{3}{2}\right)
$$

and the functions

$$
\begin{aligned}
& \psi(\tau, t)=h\left(1, \tau \hat{y}_{1}-t \hat{y}_{2}\right), \tau, t \geq 0, \\
& \sigma_{0}(\tau, t)=\left(\left\langle\varphi^{\prime}\left(\tau \hat{y}_{1}\right), \hat{y}_{1}\right\rangle,\left\langle\varphi^{\prime}\left(-t \hat{y}_{2}\right),-\hat{y}_{2}\right\rangle\right) \\
& \sigma_{1}(\tau, t)=\left(\frac{1}{\tau}\left\langle\varphi^{\prime}\left(\psi(\tau, t)^{+}\right), \psi(\tau, t)^{+}\right\rangle, \frac{1}{t}\left\langle\varphi^{\prime}\left(-\psi(\tau, t)^{-}\right),-\psi(\tau, t)^{-}\right\rangle\right) .
\end{aligned}
$$


Using the quantitative deformation theorem of Willem [26, p. 38], with the above data, we have the following result.

Proposition 18 If hypotheses $\mathrm{H}_{0}, \mathrm{H}_{1}^{\prime}$ hold and $y_{0} \in \mathrm{N}_{0}$ is the minimizer from Proposition 17 , then $y_{0} \in K_{\varphi}$.

So, under the stronger strict monotonicity condition, we can state the following multiplicity theorem for problem (1).

Proposition 19 If hypotheses $\mathrm{H}_{0}, \mathrm{H}_{1}^{\prime}$ hold, then problem (1) has at least three nontrivial solutions

$$
\begin{aligned}
& u_{0} \in \mathrm{N}_{+} \cap L^{\infty}(\Omega), u_{0}(z)>0 \text { for a.a. } z \in \Omega, m_{+}=\varphi_{+}\left(u_{0}\right), \\
& v_{0} \in \mathrm{N}_{-} \cap L^{\infty}(\Omega), v_{0}(z)<0 \text { for a.a. } z \in \Omega, m_{-}=\varphi_{-}\left(v_{0}\right), \\
& y_{0} \in \mathrm{N}_{0} \cap L^{\infty}(\Omega) \text { nodal, } m_{0}=\varphi\left(y_{0}\right) .
\end{aligned}
$$

\section{Multiple solutions under relaxed monotonicity}

In this section we drop the strict monotonicity condition $\mathrm{H}_{1}^{\prime}$ (iii) and prove a multiplicity result (three solutions theorem) with sign information for all the solutions, under the weaker set of hypotheses $\mathrm{H}_{1}$.

For this purpose, we introduce the following perturbation of the reaction

$$
f_{\ell}(z, x)=f(z, x)+\ell|x|^{r-2} x, \ell>0 .
$$

This perturbation of $f(\cdot, \cdot)$ satisfies hypotheses $\mathrm{H}_{1}^{\prime}$. We set $F_{\ell}(z, x)=\int_{0}^{x} f_{\ell}(z, s) d s$ and we consider the corresponding energy functionals $\varphi_{\ell}, \varphi_{\ell}^{ \pm}: W^{1, \theta}(\Omega) \mapsto \mathbb{R}$ defined as $\varphi, \varphi_{ \pm}$only now $F(z, x)$ is replaced by $F_{\ell}(z, x)$. Also, to these functionals we associate the corresponding Nehari manifolds $\mathrm{N}^{\ell}, \mathrm{N}_{ \pm}^{\ell}, \mathrm{N}_{0}^{\ell}$.

Proposition 20 If hypotheses $\mathrm{H}_{0}, \mathrm{H}_{1}$ hold, then there exists $\hat{\mu}>0$ such that

$$
0<\hat{\mu} \leq \varphi_{\ell}(u) \text { for all } u \in \mathrm{N}^{\ell} \text {, all } \ell \in(0,1) \text {. }
$$

Proof On account of hypotheses $\mathrm{H}_{1}$ (i), (iv), given $\varepsilon>0$, we can find $c_{17}=c_{17}(\varepsilon)>0$ such that

$$
F(z, x) \leq \frac{\varepsilon}{q}|x|^{q}+c_{17}|x|^{r} \text { for a.a. } z \in \Omega, \text { all } x \in \mathbb{R} .
$$

Let $u \in \mathrm{N}^{\ell}(\ell \in(0,1))$. From Proposition 10, we have

$$
\begin{aligned}
\varphi_{\ell}(u) & =\max _{t \geq 0} \varphi_{\ell}(t u) \\
& \geq \max _{t \geq 0}\left(\frac{t^{p}}{p} \rho_{a}(D u)+\frac{t^{q}}{q}\left(\|D u\|_{q}^{q}+\int_{\Omega} \xi(z)|u|^{q} d z\right)\right. \\
& \left.-\int_{\Omega} F(z, t u) d z-t^{r}\|u\|_{r}^{r}\right)(\text { since } 0<\ell<1) .
\end{aligned}
$$

Using (39), Proposition 5 and choosing $\varepsilon>0$ small, we obtain

$$
\begin{aligned}
\varphi_{\ell}(u) & \geq \max _{t \geq 0}\left(c_{18} t^{p}-c_{19} t^{r}\right) \text { for some } c_{18}, c_{19}>0, \text { all } t \geq 0, \\
\Rightarrow \varphi_{\ell}(u) & \geq \hat{\mu}>0(\text { recall that } p<r) .
\end{aligned}
$$


This proof is now complete.

In a similar fashion we show similar results for the functionals $\varphi_{\ell}^{ \pm}(\cdot)$.

Proposition 21 If hypotheses $\mathrm{H}_{0}, \mathrm{H}_{1}$ hold, then there exist $\hat{\mu}_{ \pm}>0$ such that

$$
\begin{aligned}
& 0<\hat{\mu}_{+} \leq \varphi_{\ell}^{+}(u) \text { for all } u \in \mathrm{N}_{+}^{\ell}, \text { all } \ell \in(0,1), \\
& 0<\hat{\mu}_{-} \leq \varphi_{\ell}^{-}(u) \text { for all } u \in \mathrm{N}_{-}^{\ell}, \text { all } \ell \in(0,1) .
\end{aligned}
$$

Now letting $\ell \rightarrow 0^{+}$we will produce constant sign and nodal solutions for problem (1) under the relaxed monotonicity condition $\mathrm{H}_{1}$ (iii).

Theorem 22 If hypotheses $\mathrm{H}_{0}, \mathrm{H}_{1}$ hold, then problem (1) has at least three nontrivial solutions

$$
\begin{aligned}
& u_{0} \in \mathrm{N}_{+} \cap L^{\infty}(\Omega), u_{0}(z)>0 \text { for a.a. } z \in \Omega, \varphi_{+}\left(u_{0}\right)=m_{+}, \\
& v_{0} \in \mathrm{N}_{-} \cap L^{\infty}(\Omega), v_{0}(z)<0 \text { for a.a. } z \in \Omega, \varphi_{-}\left(v_{0}\right)=m_{-}, \\
& y_{0} \in \mathrm{N}_{0} \cap L^{\infty}(\Omega) \text { nodal, } \varphi\left(y_{0}\right)=m_{0} .
\end{aligned}
$$

Proof Let $\ell_{n} \rightarrow 0^{+}$. From Proposition 19 we know that we can find $y_{n} \in W^{1, \theta}(\Omega)$ such that

$$
\begin{aligned}
& y_{n} \in \mathrm{N}^{\ell_{n}}(n \in \mathbb{N}), \varphi_{\ell_{n}}\left(y_{n}\right)=m_{0}^{\ell_{n}} \rightarrow \hat{m}_{0} \geq 0 \text { as } n \rightarrow \infty, \varphi_{\ell_{n}}^{\prime}\left(y_{n}\right)=0(n \in \mathbb{N}) \\
& \text { (note that }\left\{m_{0}^{\ell_{n}}\right\}_{n \in \mathbb{N}} \subseteq \stackrel{\circ}{\mathbb{R}}_{+} \text {is decreasing). }
\end{aligned}
$$

We will show that $\left\{y_{n}\right\}_{n \in \mathbb{N}} \subseteq W^{1, \theta}(\Omega)$ is bounded. Arguing by contradiction, suppose that at least for a subsequence, we have

$$
\left\|y_{n}\right\| \rightarrow+\infty
$$

We set $\hat{w}_{n}=\frac{y_{n}}{\left\|y_{n}\right\|}(n \in \mathbb{N})$. Then $\left\|\hat{w}_{n}\right\|=1$ for all $n \in \mathbb{N}$ and so we may assume that

$$
\hat{w}_{n} \stackrel{w}{\rightarrow} \hat{w} \text { in } W^{1, \theta}(\Omega) \text { and } \hat{w}_{n} \rightarrow \hat{w} \text { in } L^{r}(\Omega) .
$$

Suppose that $\hat{w} \equiv 0$ and let $k>1$. Then on account of Proposition 11, we have

$$
\begin{aligned}
& m_{0}^{\ell_{n}}= \varphi_{\ell_{n}}\left(y_{n}\right) \\
& \geq \varphi_{\ell_{n}}\left(\frac{k}{\left\|y_{n}\right\|} y_{n}\right) \\
&= \varphi_{\ell_{n}}\left(k \hat{w}_{n}\right) \\
&= \frac{k^{p}}{p} \rho_{a}\left(D \hat{w}_{n}\right)+\frac{k^{q}}{q}\left(\left\|D \hat{w}_{n}\right\|_{q}^{q}+\int_{\Omega} \xi(z)\left|\hat{w}_{n}\right|^{q} d z\right) \\
&-\int_{\Omega} F\left(z, k \hat{w}_{n}\right) d z-\ell k^{r}\left\|\hat{w}_{n}\right\|_{r}^{r} \\
& \geq \frac{k^{q}}{p} c_{20}-\int_{\Omega} F\left(z, k \hat{w}_{n}\right) d z-\ell k^{r}\left\|\hat{w}_{n}\right\|_{r}^{r} \\
& \text { for some } c_{20}>0\left(\text { since } k>1, q<p,\left\|\hat{w}_{n}\right\|=1\right) .
\end{aligned}
$$

Passing to the limit as $n \rightarrow \infty$ and recalling that $\hat{w}=0$, we obtain

$$
\hat{m}_{0} \geq \frac{k^{q}}{p} c_{20} \text { (see (40), (42)). }
$$


Since $k>1$ is arbitrary, we let $k \rightarrow+\infty$ and have a contradiction.

Now assume that $\hat{w} \neq 0$ and let $\hat{\Omega}=\{z \in \Omega: \hat{w}(z) \neq 0\}$.

Then $|\hat{\Omega}|_{N}>0$ (by $|\cdot|_{N}$ we denote the Lebesgue measure on $\mathbb{R}^{N}$ ). We have

$$
\left|y_{n}(z)\right| \rightarrow+\infty \text { for a.a. } z \in \hat{\Omega} \text {. }
$$

From (40) we have

$$
\begin{aligned}
0 & \leq \frac{m_{0}^{\ell_{n}}}{\left\|y_{n}\right\|^{p}} \\
& =\frac{\varphi_{\ell_{n}}\left(y_{n}\right)}{\left\|y_{n}\right\|^{p}} \\
& \leq \frac{1}{p} \rho_{a}\left(D \hat{w}_{n}\right)+\frac{1}{q\left\|y_{n}\right\|^{p-q}}\left(\left\|D \hat{w}_{n}\right\|_{q}^{q}+\int_{\Omega} \xi(z)\left|\hat{w}_{n}\right|^{q} d z\right)-\int_{\Omega} \frac{F\left(z, y_{n}\right)}{\left\|y_{n}\right\|^{p}} d z \\
& \leq \frac{1}{q}\left(\rho_{\theta}\left(D \hat{w}_{n}\right)+\int_{\Omega} \xi(z)\left|\hat{w}_{n}\right|^{q} d z\right)-\int_{\Omega} \frac{F\left(z, y_{n}\right)}{\left\|y_{n}\right\|^{p}} d z \text { for all } n \geq n_{1} \\
& \leq \frac{1}{q}\left(\left\|D \hat{w}_{n}\right\|_{\theta}^{q}+\int_{\Omega} \xi(z)\left|\hat{w}_{n}\right|^{q} d z\right)-\int_{\Omega} \frac{F\left(z, y_{n}\right)}{\left\|y_{n}\right\|^{p}} d z \\
& \left(\text { since }\left\|D \hat{w}_{n}\right\|_{\theta} \leq\left\|\hat{w}_{n}\right\|=1,\right. \text { see Proposition 2) } \\
& \leq \frac{1}{q} c_{21}-\int_{\Omega} \frac{F\left(z, y_{n}\right)}{\left\|y_{n}\right\|^{p}} d z \\
& \text { for some } c_{21}>0, \text { all } n \geq n_{1} \text { (see Proposition 4). }
\end{aligned}
$$

On account of hypothesis $\mathrm{H}_{1}$ (ii), we have

$$
\begin{aligned}
& \int_{\hat{\Omega}} \frac{F\left(z, y_{n}\right)}{\left\|y_{n}\right\|^{p}} d z \rightarrow+\infty, \\
\Rightarrow & \int_{\Omega} \frac{F\left(z, y_{n}\right)}{\left\|y_{n}\right\|^{p}} d z \rightarrow+\infty \\
& \left(\text { see } \mathrm{H}_{1}(\mathrm{ii})\right. \text { and use Fatou's lemma). }
\end{aligned}
$$

If in (43) we pass to the limit as $n \rightarrow \infty$ and use (44) we have a contradiction.

Therefore $\left\{y_{n}\right\}_{n \in \mathbb{N}} \subseteq W^{1, \theta}(\Omega)$ is bounded and so we may assume that

$$
y_{n} \stackrel{w}{\rightarrow} y_{0} \text { in } W^{1, \theta}(\Omega) \text { and } y_{n} \rightarrow y_{0} \text { in } L^{r}(\Omega) .
$$

From (40) we have

$$
\left\langle V\left(y_{n}\right), h\right\rangle+\int_{\Omega} \xi(z)\left|y_{n}\right|^{q-2} y_{n} h d z=\int_{\Omega} f\left(z, y_{n}\right) h d z \text { for all } h \in W^{1, \theta}(\Omega) .
$$

We choose $h=y_{n}-y_{0} \in W^{1, \theta}(\Omega)$, pass to the limits as $n \rightarrow \infty$ and use (45). We obtain

$$
\begin{aligned}
& \lim _{n \rightarrow \infty}\left\langle V\left(y_{n}\right), y_{n}-y_{0}\right\rangle=0, \\
\Rightarrow & \left.y_{n} \rightarrow y_{0} \text { in } W^{1, \theta}(\Omega) \text { (see Proposition } 3\right) .
\end{aligned}
$$

From (46) and Proposition 20, we have

$$
\begin{aligned}
& 0<\hat{\mu} \leq \varphi\left(y_{0}\right), \\
\Rightarrow & y_{0} \neq 0 \text { and so } y_{0} \in \mathrm{N} .
\end{aligned}
$$


Reasoning as in the proof Proposition 17, using Proposition 21, we show that $y_{0}^{+} \neq 0$, $y_{0}^{-} \neq 0$, hence $y_{0} \in \mathrm{N}_{0}$. Therefore we have

$$
\begin{aligned}
& y_{0} \in \mathrm{N}_{0}, \varphi^{\prime}\left(y_{0}\right)=0, \varphi\left(y_{0}\right)=m_{0}, \\
\Rightarrow & y_{0} \in \mathrm{N} \cap L^{\infty}(\Omega) \text { is a ground state nodal solution of problem (1). }
\end{aligned}
$$

Similarly, working with $\varphi_{\ell_{n}}^{+}$and $\mathrm{N}_{+}^{\ell_{n}}$ and passing to the limit as $n \rightarrow \infty$, we obtain $u_{0} \in W^{1, \theta}(\Omega)$ such that

$$
u_{0} \in \mathrm{N}_{+} \cap L^{\infty}(\Omega), \varphi^{\prime}\left(u_{0}\right)=0, u_{0}(z)>0 \text { for a.a. } z \in \Omega, \varphi\left(u_{0}\right)=m_{+}
$$

(positive ground state solution) and $v_{0} \in W^{1, \theta}(\Omega)$ such that

$$
v_{0} \in \mathrm{N}_{-} \cap L^{\infty}(\Omega), \varphi^{\prime}\left(v_{0}\right)=0, v_{0}(z)<0 \text { for a.a. } z \in \Omega, \varphi\left(v_{0}\right)=m_{-}
$$

(negative ground state solution).

Acknowledgements The research of Vicențiu D. Rădulescu and Youpei Zhang was supported by a grant of the Romanian Ministry of Research, Innovation and Digitization, CNCS/CCCDI-UEFISCDI, project number PCE 137/2021, within PNCDI III. Youpei Zhang is partially supported by the Fundamental Research Funds for the Central Universities of Central South University (no. 2019zzts211). This paper has been completed while Youpei Zhang was visiting University of Craiova (Romania) with the financial support of China Scholarship Council (no. 201906370079). Youpei Zhang would like to thank the China Scholarship Council and the Embassy of the People's Republic of China in Romania.

Open Access This article is licensed under a Creative Commons Attribution 4.0 International License, which permits use, sharing, adaptation, distribution and reproduction in any medium or format, as long as you give appropriate credit to the original author(s) and the source, provide a link to the Creative Commons licence, and indicate if changes were made. The images or other third party material in this article are included in the article's Creative Commons licence, unless indicated otherwise in a credit line to the material. If material is not included in the article's Creative Commons licence and your intended use is not permitted by statutory regulation or exceeds the permitted use, you will need to obtain permission directly from the copyright holder. To view a copy of this licence, visit http://creativecommons.org/licenses/by/4.0/.

\section{References}

1. Baroni, P., Colombo, M., Mingione, G.: Regularity for general functionals with double phase. Calc. Var. Partial Differ. Equ. 57(62), 48 (2018)

2. Brown, K.J., Wu, T.: A fibering map approach to a semilinear boundary value problem. Electron. J. Differ. Equ. No. 69, 9 pp (2007)

3. Brown, K.J., Zhang, Y.: The Nehari manifold for a semilinear elliptic equation with a sign-changing weight function. J. Differ. Equ. 193(2), 481-499 (2003)

4. Clarkc, F.: Optimization and Nonsmooth Analysis. Wiley, New York (1973)

5. Gasiński, L., Papageorgiou, N.S.: Constant sign and nodal solutions for superlinear double phase problems. Adv. Calc. Var. (2019) https://doi.org/10.1515/acv-2019-0040

6. Gasiński, L., Winkert, P.: Constant sign solutions for double phase problems with superlinear nonlinearity. Nonlinear Anal. 195, 1117399 (2020)

7. Gasiński, L., Winkert, P.: Sign changing solution for a double phase problem with nonlinear boundary condition via the Nehari manifold. J. Differ. Equ. 274, 1037-1066 (2021)

8. Ge, B., Cao, X.-F., Yuan, W.-S.: Existence of two solutions for double phase problems with a small perturbation. Appl. Anal. (2020) https://doi.org/10.1080/00036811.2021.1909725

9. Harjulehto, P., Hästö, P.: Orlicz Spaces and Generalized Orlicz Spaces. Springer, Cham (2019)

10. Lieberman, G.M.: The natural generalization of the natural conditions of Ladyzhenskaya and Ural'tseva for elliptic equations. Comm. Partial Differ. Equ. 16, 311-361 (1991)

11. Liu, W., Dai, G.: Existence and multiplicity results for a double phase problem. J. Differ. Equ. 265(9), 4311-4334 (2018) 
12. Liu, Z. Papageorgiou, N.S.: Multiple solutions with sign information for superlinear $(p, q)$-equations. Positivity (2020) https://doi.org/10.1007/s11117-021-00839-0

13. Lloyd, N.G.: Degree Theory, Cambridge Tracts in Mathematics, No. 73. Cambridge University Press, Cambridge-New York-Melbourne (1978). pp. vi+172 (ISBN: 0-521-21614-1)

14. Marcellini, P.: Regularity of minimizers of integrals of the calculus of variations with nonstandard growth conditions. Arch. Rational Mech. Anal. 105(3), 267-284 (1989)

15. Marcellini, P.: Regularity and existence of solutions of elliptic equations with $p, q$-growth conditions. J. Differ. Equ. 90(1), 1-30 (1991)

16. Mingione, G., Rădulescu, V.D.: Recent developments in problems with nonstandard growth and nonuniform ellipticity. J. Math. Anal. Appl. 501, 125197 (2021)

17. Papageorgiou, N.S., Rădulescu, V.D.: Coercive and noncoercive nonlinear Neumann problems with indefinite potential. Forum Math. 28(3), 545-571 (2016)

18. Papageorgiou, N.S., Rădulescu, V.D.: Nonlinear nonhomogeneous Robin problems with superlinear reaction term. Adv. Nonlinear Stud. 16(4), 737-764 (2016)

19. Papageorgiou, N.S., Rădulescu, V.D., Repovš, D.D.: Nonlinear Analysis-Theory and Methods. Springer Monographs in Mathematics. Springer Nature, Cham (2019)

20. Papageorgiou, N.S., Rădulescu, V.D., Repovš, D.D.: Existence and multiplicity of solutions for double phase Robin problems. Bull. Lond. Math. Soc. 52(3), 546-560 (2020)

21. Papageorgiou, N.S., Vetro, C., Vetro, F.: Solutions and positive solutions for superlinear Robin problems. J. Math. Phys. 60(10), 26 (2019)

22. Papageorgiou, N.S., Vetro, C., Vetro, F.: Multiple solutions for parametric double phase Dirichlet problems. Commun. Contemp. Math. 23(4), 18 (2021)

23. Papageorgiou, N.S., Zhang, Y.: Constant sign and nodal solutions for superlinear $(p, q)$-equations with indefinite potential and concave boundary condition. Adv. Nonlinear Anal. 10(1), 76-101 (2021)

24. Ragusa, M.A., Tachikawa, A.: Regularity for minimizers for functionals of double phase with variable exponents. Adv. Nonlinear Anal. 9, 710-728 (2020)

25. Szulkin, A., Weth, T.: In: Gao, D.Y., Motreanu, D. (eds.) The method of Nehari manifold, in Handbook of Nonconvex Analysis and Applications, pp. 597-632. International Press, Somerville (2010)

26. Willem, M.: Minimax Theorems. Birkhäuser, Boston (1996)

27. Zhikov, V.V.: Averaging of functionals of the calculus of variations and elasticity theory. Izv. Akad. Nauk SSSR Ser. Mat. 50(4), 675-710 (1986)

28. Zhikov, V.V.: On variational problems and nonlinear elliptic equations with nonstandard growth conditions. J. Math. Sci. (N.Y.) 173(5), 463-570 (2011)

Publisher's Note Springer Nature remains neutral with regard to jurisdictional claims in published maps and institutional affiliations. 